\section{New windows into the brain: technological advances in frontline neurologic diagnosis via the visual and oculomotor systems}

\author{
Jorge C Kattah ${ }^{* 1}$ \& John H Pula 2
}

The 67th American Academy of Neurology (AAN) Annual Meeting, Washington, DC, USA, 18-25 April 2015

The 67th American Academy of Neurology (AAN) Annual Meeting took place in Washington, DC, in April 2015. The first day of the meeting featured an Integrated Neuroscience Session focusing on 'Technological frontline visual and oculomotor diagnostic advances in neurologic diagnosis'. The session moderators organized presentations into two categories: visual afferent topics and vestibular/eye movement topics. In this article, we highlight the most important aspects of the research presented.

\section{Visual afferent system}

Beau Bruce presented on 'Non-mydriatic ocular fundus photography (NMOF) in neurologic emergencies'. He summarized the Emory experience with NMOF in the emergency department (ED) and demonstrated enhanced diagnostic sensitivity when using this technique for neurologic, medical, and ophthalmologic disorders. Frontline ED providers and other physicians have limited experience with non-dilated direct ophthalmoscopy, and NMOF offers a practical solution for the early detection of critical retinal and optic nerve diagnostic abnormalities in selected populations. New technology may facilitate the process even further.

The contribution of vision testing in sideline concussion evaluations for collegiate athletes was reported by Laura Balcer on behalf of her co-authors. This group investigated the King-Devick $(\mathrm{K}-\mathrm{D})$ test, a vision-based measure of rapid number naming used to complement the Sport Concussion Assessment Tool (SCAT). Worse K-D test scores were associated in their prospective cohort with worse SCAT scores. The K-D test captures visual and eye movement aspects of brain function not included in the sideline SCAT. In another report, Gardner et al. presented a video analysis of concussion in professional rugby players. The incidence was one concussion in every four games. Loss of consciousness (LOC) occurred in 30\% of cases. The remainder of patients had a vacant stare, clutching of the head, and balance problems. Seizures did not occur. All players with LOC were removed from the play and half of the remaining players continued to play in the same match. Most players with LOC returned to play the following week.

There were two presentations related to retinal and cerebral vasculature. The first reported on retinal vascular health estimated by arteriolar and venular diameter, incident proliferative retinopathy (PR), and duration of PR in Type 1 diabetic patients. This prospective study with a 25 -year

University of Illinois College of Medicine at Peoria, Illinois Neurologic Institute, Peoria, IL 61605, USA

${ }^{2}$ Northshore University Healthsystem, Evanston, IL 60201, USA

*Author for correspondence: kattahj@uicomp.uic.edu

\section{KEYWORDS}

- eye movement • neuroscience $\bullet$ oculomotor systems • vestibular movement visual afferent system • visual systems 
follow-up found retinal parameters correlated well with cognitive deficits. The association was not independent from glycemic control.

A second study presented by Diong et al. reported on a prospective study of 2506 persons aged 66-93 years who underwent brain MRI and binocular digital fundus photography from 2002-2006, as a component of the population-based AGES-Reykjavik study. Retinal microvascular changes (arteriolar narrowing, arteriovenous nicking, microaneurysms and hemorrhages), and age related macular degeneration (AMD) were correlated with cerebral microvascular bleeds (CMBs). During a mean follow up of 5.2 years, 463 people developed CMBs, 169 deep CMBs, and 294 lobar CMBs. Patients with retinal microvascular changes had a greater deep $\mathrm{CMB}$ risk. The odds ratio ranged from 1.5 to 3.5 for each specific retinal change but not for lobar CMBs. In contrast, AMD was associated with an increased risk of lobar CMBs. This study defines a positive correlation between retinal and cerebral vasculature pathology, highlighting the value of retinal vascular changes to monitor risk for CMBs.

A novel retinal imaging technique: Raman spectroscopy combined with confocal scanning laser ophthalmoscope (RS-cSLO) enabled Martinez-Lapiscina $e t$ al. to study inflammatory and neurodegenerative molecules in the inner nuclear layer of the retina, potentially preceding loss of retinal ganglion cells. This technique may detect earlier, reversible changes to target neuroprotection.

Loeb et al. reported on spectral domain OCT differences between MS and NMO. This was a prospective study of the retinal inner plexiform layer (IPL) and retinal ganglion cell (RGC) layer thickness. NMO patients showed both IPL and GCL thinning when compared with MS which preferentially affected the RGC. The NMO changes were independent of antibody seroreactivity status.

\section{Vestibular/eye movement}

In his lecture titled 'Stop radiating and start repositioning: Implementing optimal management of benign paroxysmal positional vertigo (BPPV),' Kevin Kerber reviewed epidemiologic aspects of dizziness and vertigo. Two evidence-based guidelines (American Academy of Otolaryngology - Head and Neck Surgery, and AAN) provide criteria to test, diagnose and treat BPPV. The DixHallpike test has been used for decades and the canalith repositioning maneuver is quite effective. A precise diagnosis can be made rapidly, avoiding unnecessary expensive tests. In a recent publication, Kerber reported a repositioning cure rate of approximately $80 \%$ at $24 \mathrm{~h}$, compared with only $10 \%$ of controls. Despite this large effect size, less than $10 \%$ of affected patients receive the treatment, highlighting the need to investigate educational opportunities and strategies for wide implementation. Beginning in 2008, Kerber has systematically studied the burden of illness and healthcare utilization in patients with BPPV in the ED at the Corpus Christi (BASIC) project. To begin addressing this diagnostic/treatment deficiency, the AVERT researchers will use videogoggles to record eye movements in patients with BPPV. More details on the trial and the videogoggles and their utilization were described by a second speaker.

David Newman-Toker in his lecture 'In pursuit of the "Eye EOG": eye movement-based stroke diagnosis in acute vertigo' briefly outlined the current epidemiologic data in patients with dizziness (about 4 million ED annual visits). Among them, about $5 \%$ of patients with vertigo have a stroke with high morbidity/ mortality potential. Following a decade of preliminary study, this group identified a constellation of eye movement findings in patients with vertigo which had a high sensitivity and specificity in differentiating acute vestibular syndrome (AVS) of central causes (often stroke) from more benign peripheral lesions. These are the HINTStriad and HINTS-plus. AVERT, a class 2 study, will utilize the video-goggles in all eligible AVS patients to investigate the video-goggle diagnostic accuracy in guiding management and treatment. Preliminary results have shown promising results.

Duggal et al. reported the results of brainstem auditory evoked potentials (BAEP) in 650 stroke patients, $25 \%$ of the cases with an AVS. Whereas a 48 -h brain MRI identified a stroke in $62 \%$ of these cases, head CT was negative in $92 \%$, underscoring the low sensitivity of CT in AVS. A repeat 1-month MRI showed a stroke in an additional $35 \%$ of cases. BAEP was abnormal in all initially negative stroke MRIs. Prolongation of I-III interpeak latencies was found in 68\% of cases and III-V interpeak latencies in 32\%. BAEPs were also abnormal in $30 \%$ of all AVS patients with MRI evidence of stroke. BAEPs may offer a localization diagnosis in AVS cases with stroke. 
Jorge Kattah reported on a V4 vertebral artery signal change (the 'target sign') in a retrospective study of prospectively recruited AVS patients. Axial T2 MRI scans indicated impaired blood flow or occlusion in 31/68 stroke patients but only $4 / 67$ vestibular neuritis patients. The odds were 20.29 greater for stroke when the target sign was present.

Two novel studies were discussed in this session pertaining rapid triaging of stroke patients to expedite administration of rtPA. The first from Padrick et al. found that the assessment of the NIH stroke scale evaluated via Tablet-Based Mobile Telestroke during ambulance transport correlated well with face to face assessments, leading potentially to faster thrombolytic administration. In a second presentation, Grotta reported an on-going study investigating the benefits of Stroke Treatment Delivered using a Mobile Stroke Unit (MSU). The MSU has a vascular neurologist (VN), a registered nurse, and a CT technologist. The MSU staff evaluates rtPA administration eligibility criteria and enrolls patients. Simultaneously, the process is monitored with Telemedicine with a remote VN making an independent recommendation. All patients are transferred to the Stroke Comprehensive Unit. The MSU process is compared with the standard EMS process. This study began in 2014 and administered rtPA in 13 patients within an average time of $26 \mathrm{~min}$ on scene, 11 patients were enrolled but did not receive rtPA (four of them with cerebral hemorrhage and seven with other nonstroke diagnoses).

In conclusion, the AAN session on technological advances in the visual and oculomotor systems presented a wide variety of novel and exciting research and garnered excitement from the audience about future discoveries yet to come [1-5].

\section{Financial \& competing interests disclosure}

The authors have no relevant affliations or financial involvement with any organization or entity with a financial interest in or financial conflict with the subject matter or materials discussed in the manuscript. This includes employment, consultancies, honoraria, stock ownership or options, expert testimony, grants or patents received or pending, or royalties.

No writing assistance was utilized in the production of this manuscript.

\section{References}

1 Bruce BB, Biousse V, Newman NJ. Nonmydriatic ocular fundus photography in neurologic emergencies. JAMA Neurol. 72(4), 455-459, PMID: 25665183 (2015).

2 Leong DL, Balcer LJ, Galetta SL, Evan G, Gimre M, Watt D. The King-Devick Test for sideline concussion screening in collegiate football. J. Optom. 8, 131-139 (2015).

C, Cotch MF, Sigurdsson S et al. Microvascular lesions in the brain and retina: The AGES Reykjavik Study. Ann. Neurol. 65, 569-576 (2009).

4 Kerber K, Helmchen C. Benign paroxysmal positional vertigo. New opportunities but still old challenges. Neurology 78, 3154-3156 (2012).

5 Newman Toker DE, Saber Tehrani AS, Mantokoudis G et al. Quantitative videooculography to help diagnose in acute vertigo and dizziness: toward an ECG of the eyes. Stroke 44, 1158-1161 (2013). 